\title{
Infección por Strongyloides stercoralis: estudio epidemiológico, clínico, diagnóstico y terapéutico en 30 pacientes
}

Marcelo Corti, María F. Villafañe, Norberto Trione, Daniel Risso, Juan Carlos Abuín y Omar Palmieri

\section{Infection due to Strongyloides stercoralis: epidemiological, clinical, diagnosis findings and outcome in 30 patients}

Background: Strongyloides stercoralis is a nematode parasite, which is endemic in tropical and subtropical regions. Infection usually remains asymptomatic, but in immunocompromised hosts severe and life-threatening manifestations such as hyperinfection syndrome and disseminated disease might occur. Methods: We retrospectively analyzed the epidemiological and clinical characteristics, including HIV co-infection, microbiological findings, and outcome in 30 patients with strongyloidiasis, who attended in the Infectious Diseases F. J. Muñiz Hospital in Buenos Aires from January 2004 to December 2008. Results: The study included 20 men and 10 women with an average age of 33 years. HIV co-infection was present in 21 patients $(70 \%)$ with a median CD4 T cell count of 50 cells $/ \mathrm{mm}^{3}$ (range 7-355) (average 56 cells $/ \mathrm{mm}^{3}$ ). Among HIV negative patients the following comorbidities were detected: tuberculosis $(\mathrm{n}=3)$ and chronic alcoholism, leprosy treated with corticosteroids, immunosuppressive treatment for psoriasis, and diabetes mellitus (each in one patient). Two patients did not have any predisposing diseases or immunosuppressive treatments. Seventeen patients presented with diarrhea and were classified as chronic intestinal strongyloidiasis (57\%), asymptomatic infection with peripheral eosinophilia was diagnosed in 7 (23\%), and 6 patients $(20 \%)$ developed hyperinfection syndrome. Seventeen patients $(57 \%)$ presented peripheral eosinophilia. Diagnosis was achieved by direct visualization of larvae in feces by Baermann technique $(n=20)$, by multiple stool smears examinations $(n=2)$, by combination of both $(n=1)$, by visualization of the filariform larvae in duodenal fluid and stool $(\mathrm{n}=1)$, and in fecal and bronchoalveolar lavage specimens $(\mathrm{n}=6)$. Overall mortality in this series was $20 \%(6 / 30)$. There was no significant correlation between age and mortality. A significant inverse correlation between the survival rate and CD4 T-cell count as well as eosinophilia was observed. There was also a significant correlation between HIV co-infection and mortality. Twenty-two patients responded favorably to treatment with ivermectin.

Key words: Strongyloides stercoralis, strongyloidiasis, HIV, AIDS.

Palabras clave: Strongyloides stercoralis, estrongiloidiasis, VIH, SIDA.

\section{Introducción}

S trongyloides stercoralis es un nematodo endémico en áreas tropicales y templadas de todo el mundo. geo-helminto es su ciclo vital complejo que le permite provocar auto-infestación en el hospedero. En la mayoría de los casos la infección permanece asintomática, en especial en las áreas endémicas. Sin embargo, bajo ciertas condiciones de inmunodepresión, S. stercoralis puede provocar cuadros graves y a veces mortales que incluyen formas de hiperinfestación o diseminadas ${ }^{1,2}$. Es una parasitosis de difícil diagnóstico, ya que el mismo requiere de la visualización directa de las formas larvarias del parásito y no existe una prueba que se asocie con $100 \%$ de sensibilidad. En consecuencia, es muy importante para el médico clínico, en particular el infectólogo, la sospecha diagnóstica, en especial en aquellos grupos de pacientes con causas predisponentes para las manifestaciones graves de esta enfermedad ${ }^{3}$. En este estudio retrospectivo se describen las características epidemiológicas, clínicas, condiciones predisponentes y la evolución de 30 pacientes con diagnóstico de estrongiloidiasis.

\section{Métodos}

Se analizaron de manera retrospectiva 30 pacientes con diagnóstico de estrongiloidiasis asistidos en el Hospital de Referencia para Enfermedades Infecciosas del gobierno
Hospital de Enfermedades Infecciosas Francisco J. Muñiz, Buenos Aires, Argentina

Recibido: 25 de julio de 2010 Aceptado: 4 de abril de 2011

Correspondencia a: Marcelo Corti marcelocorti@fibertel.com.ar 
de la Ciudad Autónoma de Buenos Aires entre enero de 2004 y diciembre de 2008. Se incluyeron todos aquellos pacientes asistidos durante el periodo señalado, que presentaron diarrea o eosinofilia periférica $(>5 \%)$ en los que se identificaron larvas de $S$. stercoralis en exámenes de heces, esputo, lavado bronco-alveolar (LBA), líquido duodenal o biopsias de la mucosa duodenal. Se consideraron las características epidemiológicas, clínicas, la condición serológica frente al virus de la inmunodeficiencia humana (VIH), la presencia de otras co-morbilidades predisponentes, los hallazgos de laboratorio, la evolución y la respuesta al tratamiento. En el caso de aquellos pacientes con infección por VIH se consideró la posibilidad de otras infecciones oportunistas o neoplasias marcadoras del SIDA y el nivel de linfocitos T CD4+ al momento del diagnóstico de la estrongiloidiasis. La parasitosis se diagnosticó mediante la visualización de las larvas rhabditoides en muestras seriadas de materia fecal tamizadas, las que fueron observadas en fresco con microscopia directa. En el caso de que el análisis de la primera muestra hubiera

Tabla 1. Características demográficas, clínicas, inmunológicas y evolutivas de los pacientes co-infectados por VIH y S. stercoralis.

\begin{tabular}{|c|c|c|c|c|c|}
\hline $\begin{array}{l}\text { Edad } \\
\text { (años) }\end{array}$ & Sexo & $\begin{array}{c}\text { Linfocitos T CD4+ } \\
\left.\text { (céls } / \mathrm{mm}^{3}\right)\end{array}$ & Forma clínica & $\begin{array}{c}\text { Eosinofilia } \\
\text { periférica } \\
(\%)\end{array}$ & Supervivencia \\
\hline 47 & M & 124 & Asintomática & 48 & Sí \\
\hline 25 & $\mathrm{~F}$ & 7 & Intestinal crónica & 0 & Óbito \\
\hline 41 & M & 12 & Hiperinfestación & 4 & Sí \\
\hline 29 & M & 58 & Asintomática & 16 & Óbito \\
\hline 24 & M & 10 & Intestinal crónica & 0 & Sí \\
\hline 55 & $\mathrm{~F}$ & 23 & Hiperinfestación & 1 & Óbito \\
\hline 29 & M & 36 & Asintomática & 9 & Sí \\
\hline 26 & $\mathrm{~F}$ & 148 & Intestinal crónica & 7 & Sí \\
\hline 50 & $\mathrm{~F}$ & 335 & Intestinal crónica & 27 & Sí \\
\hline 38 & M & 154 & Intestinal crónica & $N / R$ & Óbito \\
\hline 33 & M & 47 & Hiperinfestación & 0 & Óbito \\
\hline 39 & M & 210 & Intestinal crónica & 24 & Sí \\
\hline 27 & $\mathrm{~F}$ & 50 & Intestinal crónica & 2 & Sí \\
\hline 38 & M & 258 & Intestinal crónica & $N / R$ & Sí \\
\hline 28 & M & $N / R$ & Hiperinfestación & 9 & Sí \\
\hline$N / R$ & M & $N / R$ & Intestinal crónica & 1 & Sí \\
\hline$N / R$ & M & 50 & Intestinal crónica & 2 & Óbito \\
\hline 38 & M & 47 & Hiperinfestación & 0 & Sí \\
\hline 33 & M & 50 & Intestinal crónica & 0 & Sí \\
\hline 29 & M & 50 & Intestinal crónica & 2 & Sí \\
\hline 10 & $\mathrm{~F}$ & $N / R$ & Asintomática & 6 & Sí \\
\hline
\end{tabular}

resultado negativa y los antecedentes clínicos y epidemiológicos hicieran sospechar la presencia de esta parasitosis, se procedió a reiterar el examen copro-parasitológico con la técnica de concentración de Baermann con el fin de incrementar el rendimiento diagnóstico. Esta técnica permite separar selectivamente las larvas, lo que es muy útil para su aislamiento e identificación ${ }^{4}$. La obtención de material duodenal mediante aspirado o biopsia sólo se efectuó en aquellos pacientes con sospecha de otras co-infecciones. En los pacientes con manifestaciones clínicas compatibles con estrongiloidiasis diseminada y síntomas respiratorios se analizaron muestras de esputo y LBA en fresco y con las tinciones de Gram, ZiehlNeelsen y Grocott. En los pacientes con manifestaciones neurológicas se analizaron muestras del centrifugado del líquido cefalorraquídeo (LCR) para el examen directo en fresco y con las tinciones de Gram y Ziehl-Neelsen. También se evaluó el recuento de eosinófilos en sangre periférica. Para la categorización de las formas clínicas, se clasificó a los pacientes en asintomáticos, con formas intestinales crónicas, de hiperinfestación y diseminadas. El diagnóstico de hiperinfestación se consideró por la presencia de manifestaciones clínicas graves asociadas con el compromiso de órganos habitualmente involucrados en el ciclo vital del parásito (ciclo de Looss) ${ }^{5}$ como el tracto gastrointestinal y el pulmón, debido a una autoreinfestación masiva, lo que origina una alta carga larvaria en heces y esputo. La forma diseminada de la enfermedad se definió por la presencia de larvas filariformes en sitios ectópicos fuera del intestino o del pulmón, tales como ganglios linfáticos, hígado, bazo, tiroides, endocardio, riñones, pleuras, cerebro y meninges, con la posibilidad de llevar bacilos gramnegativos a esos órganos. Para el análisis estadístico se utilizó la prueba de t de Student con la que se determinaron las diferencias entre las muestras y se calculó el intervalo de confianza y el error estándar. Para medir la relación entre variables cuantitativas se utilizó el coeficiente de correlación de Pearson. Los cálculos se efectuaron con el programa Microsoft Excel. Se consideró significativo un valor de $\mathrm{p}<0,05$.

\section{Resultados}

De los 30 pacientes que se incluyeron en la evaluación, 20 fueron hombres (67\%) y 10 mujeres (33\%). La mediana de edad (excluyendo a los pacientes pediátricos) fue de 33 años; e incluyendo a los niños este parámetro fue de 32 años. La media de edad \pm DE (excluyendo a los pacientes pediátricos) fue de 36,29 $\pm 12,55$ años e incluyendo a los niños fue de $34,37 \pm 13,87$ años.

La serología para VIH fue reactiva en 21 pacientes $(70 \%) ; 15$ fueron varones $(71 \%)$ y seis mujeres $(29 \%)$ (Tabla 1). Entre los nueve restantes, seronegativos para 
VIH, hubo cinco mujeres y cuatro varones (Tabla 2). Las conductas de riesgo para la infección por VIH fueron: relaciones heterosexuales no protegidas en nueve pacientes $(43 \%)$, adicción a drogas intravenosas en 7 (33\%), relaciones homosexuales no protegidas en cuatro (19\%) y transmisión vertical en el restante $(5 \%)$. La mediana de linfocitos T CD4+ de este subgrupo de pacientes al momento del diagnóstico de la parasitosis fue de 50 céls/ $\mathrm{mm}^{3}$ (rango 7 a 335) (Tabla 1).

En los pacientes seronegativos para VIH, se comprobaron las siguientes co-morbilidades: tuberculosis (TBC) en tres casos; y un caso de cada una de las siguientes afecciones: alcoholismo crónico, diabetes mellitus, reacción lepromatosa tratada con altas dosis de corticosteroides, y otro con psoriasis en tratamiento inmunosupresor con metotrexato y corticosteroides. En los dos pacientes restantes no se reconocieron enfermedades ni terapias predisponentes.

En relación con las formas clínicas, se diagnosticaron 17 pacientes con enfermedad intestinal crónica (57\%), siete casos de infección asintomática $(23 \%)$ en los que el diagnóstico se sospechó por la eosinofilia periférica; un paciente de este grupo presentó una bacteriemia por Pseudomonas aeruginosa.

Hubo seis casos de síndrome de hiperinfestación $(20 \%)$ diagnosticados por la identificación de larvas en la materia fecal y secreciones broncopulmonares; cinco de ellos (83\%) se presentaron en el grupo de pacientes infectados con VIH (Tabla 3). El restante se diagnosticó en un paciente seronegativo para $\mathrm{VIH}$, con antecedentes de alcoholismo, tabaquismo y TBC pulmonar. Del los 21 pacientes con infección por $\mathrm{VIH}$, cinco $(24 \%)$ presentaron síndrome de hiperinfestación. Todos tenían diagnóstico previo de SIDA con recuentos de linfocitos T CD4+ menor a 50 céls $/ \mathrm{mm}^{3}$ y habían recibido corticoterapia prolongada debido a una neumonía por Pneumocystis jiroveci, criptococosis diseminada con hipertensión endocraneal y toxoplasmosis cerebral en tres casos y quimioterapia por sarcoma de Kaposi en los dos restantes. De los 28 pacientes de la serie en que se contó con los resultados del porcentaje de eosinófilos en sangre periférica, 17 (57\%) presentaron eosinofilia periférica (rango 6 a 48\%); de éstos, $16(94 \%)$ respondieron favorablemente a la terapia antiparasitaria, con un solo óbito en este grupo. Por el contrario, entre los 11 pacientes que no tuvieron respuesta de eosinófilos en sangre periférica, sólo seis (54\%) respondieron al tratamiento, constatándose cinco óbitos (45\%) en este grupo. La prueba de $t$ de Student para determinar las diferencias entre la respuesta de eosinófilos y la letalidad (incluyendo los 28 pacientes en que estos datos estuvieron disponibles) fue significativa con un valor de $\mathrm{p}<0,001$. El coeficiente de correlación de Pearson para las mismas variables fue de $r=-0,28$ (significativo).

En 22 casos el diagnóstico se efectuó por la visualiza-

\begin{tabular}{|ccccc|}
\hline \multicolumn{5}{|c|}{$\begin{array}{c}\text { Tabla 2. Características demográficas, clínicas, inmunológicas y evolutivas } \\
\text { de los pacientes seronegativos para VIH con estrongiloidiasis }\end{array}$} \\
\begin{tabular}{|cccc} 
Edad \\
(años)
\end{tabular} & Sexo & Forma clínica & $\begin{array}{c}\text { Eosinofilia periférica } \\
(\%)\end{array}$ & Supervivencia \\
\hline 28 & F & Asintomática & 20 & Sí \\
31 & M & Asintomática & 12 & Sí \\
\hline 65 & M & Hiperinfestación & 17 & Sí \\
\hline 36 & M & Asintomática & 15 & Sí \\
N/R & F & Intestinal crónica & 18 & Sí \\
\hline 16 & M & Intestinal crónica & 10 & Sí \\
\hline 66 & F & Intestinal crónica & 12 & Sí \\
\hline 12 & F & Intestinal crónica & 26 & Sí \\
\hline N/R & M & Intestinal crónica & 9 & Sí \\
\hline N/R: no registrado. & & & \\
\hline
\end{tabular}

Tabla 3. Características demográficas, clínicas, inmunológicas y evolutivas de los pacientes con síndrome de hiperinfestación por Strongyloides stercoralis

\begin{tabular}{|c|c|c|c|c|c|c|}
\hline $\begin{array}{l}\text { Edad } \\
\text { (años) }\end{array}$ & Sexo & VIH & $\begin{array}{c}\text { Linfocitos } \\
\text { T CD4+ } \\
\left.\text { (céls/mm } / \mathrm{mm}^{3}\right)\end{array}$ & $\begin{array}{l}\text { Enfermedades } \\
\text { marcadoras previas }\end{array}$ & $\begin{array}{c}\text { Eosinofilia } \\
\text { periférica } \\
(\%)\end{array}$ & $\begin{array}{l}\text { Supervi- } \\
\text { vencia }\end{array}$ \\
\hline 41 & M & $(+)$ & 12 & SK diseminado & 4 & Sí \\
\hline 55 & $\mathrm{~F}$ & $(+)$ & 23 & Criptococosis diseminada & 1 & Óbito \\
\hline 33 & M & $(+)$ & 47 & Neumonía P. jiroveci & 0 & Óbito \\
\hline 28 & M & $(+)$ & $N / R$ & Toxoplasmosis cerebral & 9 & Sí \\
\hline 38 & M & $(+)$ & 47 & SK diseminado & 0 & Sí \\
\hline 65 & M & $(-)$ & 739 & Tuberculosis pulmonar $\left({ }^{*}\right)$ & 17 & Sí \\
\hline
\end{tabular}

ción directa de las larvas en muestras de heces en fresco, mediante la técnica de concentración de Baermann. El diagnóstico también se efectuó por el examen coproparasitológico seriado en dos pacientes, por ambos métodos en un caso, en líquido duodenal y materia fecal en un caso y por la identificación de larvas en materia fecal y secreciones respiratorias en seis pacientes. La letalidad global en esta serie fue de $20 \%$ (6/30). Los seis pacientes fallecidos tenían diagnóstico de SIDA avanzado, y de éstos, sólo uno presentó una respuesta de eosinófilos del $16 \%$. El resto de los fallecidos evolucionó con aneosinofilia. El coeficiente de correlación de Pearson entre la edad y la supervivencia fue de $r=-0,06$ (no significativo). El coeficiente de correlación entre el recuento de CD4 y la letalidad, incluyendo 18 de los 21 pacientes infectados por VIH en los que este dato estuvo disponible, fue de $r=-0,28$ (significativo) y el valor de $\mathrm{p}$ (estimado por t de Student) fue de 0,03 (significativo). Finalmente, el coeficiente de correlación de Pearson entre la seropositividad para VIH y la letalidad fue de $r=1$ (significativo). 
Veinticuatro pacientes respondieron a la terapia antiparasitaria y evolucionaron favorablemente. El fármaco utilizado en el tratamiento de todos los pacientes de esta serie fue ivermectina, en dosis única de $0,2 \mathrm{mg} / \mathrm{kg}$ que fue repetida cada 14 días en los pacientes con infección por VIH, hasta alcanzar la reconstitución inmune con la terapia anti-retroviral.

\section{Discusión}

La estrongiloidiasis es una parasitosis endémica en países de clima tropical o subtropical, donde las condiciones climáticas de humedad y temperatura permiten el desarrollo y la supervivencia de S. stercoralis ${ }^{6}$. La población mundial infectada asciende aproximadamente a 100 millones de personas y la pandemia de SIDA, así como otras causas de inmunodepresión, ha incrementado la frecuencia de formas graves de la enfermedad. La infección inicial es, por lo general, asintomática y lo habitual es la tendencia a la cronicidad. En más de la mitad de los pacientes el único hallazgo para el diagnóstico presuntivo es la eosinofilia en sangre periférica. Ésta es, por lo general, de 5 a 10\% y se comprueba en 80 a $90 \%$ de los $\operatorname{casos}^{6}$. En el síndrome de hiperinfestación y en las formas diseminadas es frecuente la ausencia de eosinofilia ${ }^{7}$. Cuando existen síntomas, éstos suelen ser esporádicos y de larga duración. Comprometen con más frecuencia el tubo digestivo (epigastralgia, dispepsia, diarrea y malabsorción crónica), el aparato respiratorio (tos y broncoespasmo) y la piel (prurito). La infección por S. stercoralis incluye cinco síndromes clínicos: portación; infección aguda con síndrome de Loeffler; infección intestinal crónica con diarrea; síndrome de hiperinfestación y enfermedad diseminada ${ }^{8}$.

La forma de presentación y la evolución de esta infección están determinadas por la interacción entre el hospedero y el parásito. La activación de la respuesta inmune celular de linfocitos T helper 2 (Th2), de la respuesta inmune humoral o la alteración de las barreras mucosas favorecen la transformación de la larva rhabditoide en filariforme infestante, lo cual va seguido de su migración por vía sanguínea desde el intestino delgado al pulmón; allí perfora el alvéolo y cae en el espacio aéreo, asciende por los bronquios, tráquea, faringe, esófago y llega al intestino, donde la larva se transforma en hembra adulta partenogenética y comienza la postura de huevos embrionados, de los que rápidamente eclosiona una larva rhabditoide. Así, en forma continua se repite el ciclo, lo que origina el síndrome de hiperinfestación. A pesar del gran número de personas co-infectadas con VIH y S. stercoralis, los casos descritos de hiperinfestación son inusuales en la literatura médica; una revisión efectuada en el año 2004 sólo comprobó menos de 30 casos de hiperinfestación asociada a infección por VIH. En estos pacientes debe considerarse también como factor de riesgo la corticoterapia en altas dosis utilizada para el tratamiento de la neumonía por $P$. jiroveci, la encefalitis por Toxoplasma gondii y como parte de la quimioterapia para los linfomas no Hodgkin. Por este motivo, en aquellos pacientes que van a recibir terapia prolongada con corticosteroides o presentan otras co-morbilidades como alcoholismo o diabetes mellitus, deberá descartarse la infestación por este geo-helminto, en especial si se detecta eosinofilia periférica. La infección por el virus linfotrópico-T humano tipo-1 (HTLV-1) se asocia con una elevada prevalencia de infección por $S$. stercoralis. En estos enfermos suele ser frecuente la falta de respuesta al tratamiento antiparasitario y el síndrome de hiperinfestación. Este podría explicarse porque la infección crónica por HTLV-1 afectaría la respuesta inmune específica frente a $S$. stercoralis y reduciría la respuesta al tratamiento. Además, la infección por S. stercoralis, modifica la historia natural de la infección por el retrovirus HTLV-1. De esta manera, los pacientes infectados por HTLV-1 que desarrollan una leucemia de células T del adulto y que están co-infectados por S. stercoralis son significativamente más jóvenes que aquellos sin infección por el geo-helminto. Esto sugiere que la coinfección podría acortar el largo período que transcurre habitualmente hasta el desarrollo de la neoplasia ${ }^{4}$. En la serie que se analiza no se investigó la co-infección por HTLV- 1 debido a la muy baja prevalencia de la misma en nuestro país $(0,05 \%$ en bancos de sangre; rango 0,03 a $0,16 \%$, de acuerdo con la región geográfica considerada) ${ }^{10}$. En las formas diseminadas de la enfermedad ${ }^{9}$, como ya fue expresado, la presencia de la larva se observa en casi todos los órganos y tejidos. En su migración puede llevar bacilos gramnegativos desde el intestino y originar meningitis, neumonía y septicemia. La letalidad es de $\sim 15 \%$ en la hiperinfestación y se incrementa a $85 \%$ en las formas diseminadas ${ }^{11}$.

En la serie que se presenta, la letalidad global fue de $20 \%$ y estuvo fuertemente asociada a la infección por VIH con bajos recuentos de linfocitos T CD4+ y a la aneosinofilia periférica. La elevada letalidad en estos casos suele relacionarse con infecciones bacterianas sobreagregadas. El objetivo principal del tratamiento de esta parasitosis consiste en evitar el síndrome de hiperinfestación, en especial en pacientes inmunocomprometidos. Este criterio incluye la erradicación del parásito y no sólo la respuesta sintomática. En la actualidad el tratamiento de elección es ivermectina, a la dosis de $0,2 \mathrm{mg} / \mathrm{kg}(200 \mu \mathrm{g} / \mathrm{kg})$ en una sola toma, durante dos días. En las formas graves, algunos autores proponen prolongar el tratamiento a tres días ${ }^{12}$. Además, es recomendable repetir la dosis luego de dos semanas para asegurar la erradicación de las larvas, ya que este es el tiempo que dura un ciclo de autoinfestación. Su eficacia y tolerancia son excelentes; entre 80 y $100 \%$, 
respectivamente ${ }^{13}$. En la serie que se presenta, todos los pacientes fueron tratados con ivermectina con una eficacia de $76 \%$ discretamente inferior a la referida por la literatura científica ${ }^{12}$. Es probable que en los pacientes con SIDA avanzado sea necesario repetir nuevas dosis de este fármaco hasta alcanzar la reconstitución inmunológica asociada con la terapia anti-retroviral ${ }^{14}$.

La gravedad de las formas de hiperinfestación y de las diseminadas hace necesaria la búsqueda de este helminto en las heces de pacientes inmunocomprometidos, en especial los sometidos a corticoterapia prolongada, terapia inmunosupresora posterior a trasplante de órganos sólidos o precursores hematopoyéticos y SIDA avanzado ${ }^{8,14}$. De todos los fármacos inmunosupresores, los corticosteroides son los más utilizados y que con mayor frecuencia se asocian con la transformación de la estrongiloidiasis intestinal crónica en formas diseminadas o el síndrome de hiperinfestación. La letalidad de este último alcanza a 50\% de los pacientes a pesar del tratamiento antiparasitario $^{15}$. El tamaño de la muestra analizada en esta serie de un único centro asistencial, el Hospital de Referencia para Enfermedades Infecciosas de la República Argentina, limitó el poder estadístico del estudio. Sin embargo, pudo establecerse una diferencia significativa entre la mayor letalidad y la ausencia de respuesta periférica de eosinófilos, la condición de VIH positivo y el bajo recuento de linfocitos T CD4 +.

En conclusión, la infección por S. stercoralis es con frecuencia subestimada; sin embargo debe considerarse una enfermedad emergente, tanto en áreas industrializadas como subdesarrolladas del planeta. Su diagnóstico debe considerarse en todos aquellos pacientes con eosinofilia periférica y con factores de riesgo epidemiológico.

Agradecimiento. Los autores agradecen a Leonardo Gilardi por su colaboración en el análisis estadístico de los resultados de esta serie.

\section{Resumen}

Antecedentes: Strongyloides stercoralis, parásito endémico de áreas tropicales y subtropicales del planeta, en sujetos inmunodeprimidos puede cursar con formas graves y aun mortales como el síndrome de hiperinfestación y la enfermedad diseminada. Métodos: Análisis retrospectivo de las características epidemiológicas, manifestaciones clínicas, co-infección por virus de inmunodeficiencia humana (VIH), hallazgos microbiológicos y evolución de 30 pacientes con estrongiloidiasis, atendidos en el Hospital de Enfermedades Infecciosas F. J. Muñiz de Buenos Aires, entre enero 2004 y diciembre 2008. Resultados: Se incluyeron en la evaluación 20 hombres y 10 mujeres con una mediana de edad de 33 años. Co-infección por VIH hubo en 21 pacientes (70\%); la mediana de linfocitos T CD4+ en ellos al momento del diagnóstico de la parasitosis fue de 50 céls $/ \mathrm{mm}^{3}$ (rango 7 a 355), (media de 56 céls $/ \mathrm{mm}^{3}$ ). En los pacientes seronegativos para VIH, se comprobaron las siguientes co-morbilidades: tuberculosis (n: 3) y un caso de cada una de las siguientes afecciones: alcoholismo crónico, diabetes mellitus, reacción lepromatosa bajo corticoterapia, y psoriasis en tratamiento inmunosupresor. Hubo dos pacientes sin aparentes enfermedades de base. Diecisiete pacientes presentaron enfermedad intestinal crónica con diarrea (57\%), era asintomática y fue sospechada por la eosinofilia periférica (n: 7, 23\%) y se clasificó como síndrome de hiperinfestación (n: 6, 20\%) diagnosticado por la identificación de larvas en la materia fecal y secreciones broncopulmonares. Diecisiete pacientes (57\%) presentaron eosinofilia periférica. El diagnóstico se efectuó por la visualización directa de las larvas en muestras de heces en fresco mediante la técnica de concentración de Baerman (n: 20); por el examen copro-parasitológico seriado (n: 2) y por ambos métodos (n: 1); en líquido duodenal y materia fecal (n: 1) y por la identificación de larvas en materia fecal y secreciones respiratorias (n: 6). Letalidad global: 20\% (6/30). Los pacientes con eosinofilia tuvieron una menor letalidad que aquellos sin esta respuesta $(\mathrm{p}<0,001)$. No hubo correlación estadística entre la edad y la supervivencia. Sí fue significativa la correlación entre el recuento de CD4 y la letalidad, incluyendo 18 de los 21 pacientes seropositivos para VIH (p: 0,03). Finalmente, la correlación seropositividad para VIH y letalidad también fue significativa. Veintidós pacientes respondieron a la terapia antiparasitaria con ivermectina y evolucionaron favorablemente.

\section{Referencias}

1.- Mahmoud A A. Strongyloidiasis. Clin Infect Dis 1996; 23: 949-52.

2.- Heyworth M F. Parasitic diseases in immunocompromised hosts: cryptosporidiasis, isosporidiasis and strongyloidiasis. Gastroenterol Clin North Am 1996; 25: 691-707.

3.- Liu L, Weller P. Strongyloidiasis and other intestinal nematode infections. Infect Dis Clin
North Am 1993; 3: 655-82.

4.- Keiser P B, Nutman T B. Strongyloides stercoralis in the immunocompromised population. Clin Microbiol Rev 2004; 17 : 208-17.

5.- Looss A. Die Wanderung der Ancylostomumund-Strongyloides-larven von der Haut nach dem Darm. Berne, Comptes Rendus 6me Congres International Zoologie, 1904, pp. 225-33.
6.- Pardo Romero G, Rodríguez Rodríguez R, Campillos Páez MT. Strongyloides stercoralis: factores de riesgo para estrongiloidiosis diseminada. Med Clin (Barc) 2003; 121: 662-4.

7.- Genta R M, Milles P, Fields K. Opportunistic Strongyloides stercoralis infection in lymphoma patients: report of a case and review of the literature. Cancer 1989; 63: 1407-11.

8.- Marcos L A, Terashima A, DuPont H L, Gotuzzo E. Strongyloides hyperinfection 
syndrome: an emerging global infectious disease. Trans R Soc Trop Med Hyg 2008; 102 : 314-8.

9.- Concha R, Harrington Jr W, Rogers AI. Intestinal strongyloidiasis: recognition, management, and determinants of outcome. J Clin Gastroenterol 2005; 39: 203-11.

10.- Biglione M M, Astarloa L, Salomón H E and the Referent HTLV I/II Argentina Group. AIDS Res Hum Retroviruses 2005; 21: 1-4.
11.- Vadlamudi R S, Chi D S, Krishnaswamy G. Intestinal strongyloidiasis and hyperinfection syndrome. Clin Mol Allergy 2006; 30: 4-8.

12.- Nozais J P, Thellier M, Datry A, Danis M. Disseminated strongyloidiasis. Presse Med 2001; 30: 813-8.

13.- Pardo Romero G, Rodríguez Rodríguez R, Campillos Páez MT. Stongyloides stercoralis: factores de riesgo para estrongiloidosis diseminada. Med Clin (Barc) 2003; 121: 662-4.

14.- Roxby A C, Gottlieb G S, Limaye A P. Strongyloidiasis in transplant patients. Clin Infect Dis 2009; 49: 1141-23.

15.- Al Maslamani M A, Al Soub H A, Al Khal A L, Al Bozom I A, Abu Khatatab M J, Chacko K C. Strongyloides stercoralis hyperinfection after corticosteroid therapy: a report of two cases. Ann Saudi Med 2009; 29: 397-401. 